\title{
Increased skeletal muscle expression of VEGF induced by massage and exercise
}

\author{
Waldemar Andrzejewski ${ }^{1,2}$, Krzysztof Kassolik ${ }^{1,2}$, Christopher Kobierzycki, ${ }^{1,}$, \\ Jedrzej Grzegrzolka ${ }^{3}$, Katarzyna Ratajczak-Wielgomas ${ }^{3}$, Karolina Jablonska ${ }^{3}$, \\ Tomasz Halski ${ }^{2}$, Piotr Dziegiel ${ }^{1,3}$, Bohdan Gworys ${ }^{4}$, Marzenna Podhorska-Okolow ${ }^{3}$ \\ ${ }^{1}$ Department of Physiotherapy, University School of Physical Education, Wroclaw, Poland \\ ${ }^{2}$ Public Higher Medical Professional School, Opole, Poland \\ ${ }^{3}$ Department of Histology and Embryology, Wroclaw Medical University, Wroclaw, Poland \\ ${ }^{4}$ Department of Anatomy, Wroclaw Medical University, Wroclaw, Poland
}

\begin{abstract}
Introduction. Numerous investigations have been carried out to describe the role of massage in preparing for and restoring efficiency after physical exercise. Furthermore, vascular endothelial growth factor (VEGF) enhances blood vessel growth, and in effect contributes to the regeneration of tissues. Since its expression in active skeletal muscles has not been yet determined, the aim of this study was to investigate whether muscle massage performed before and during running exercise affects the expression of VEGF-A in muscles.

Material and methods. The study was carried out on 75 adult Buffalo rats subjected to running exercise training for 10 weeks. Rats were massaged prior (group PM) or during exercise (group M) or were not massaged (group C). The massage consisted of spiral movements along the plantar surface of flexor digitorum brevis muscle. After 1, 3, 5,7 and 10 week of training, five rats from every group were anesthetized and immunohistochemistry, Western blot, and PCR analyses were performed on obtained muscle tissue to determine VEGF-A expression.

Results. After the first week of training, a significant increase of VEGF-A gene expression analyzed by qPCR in muscle tissue was observed in the PM group, whereas in the third week, the predominant growth of studied marker was seen in the M group. Increased VEGF-A expression on the protein level was observed in both massaged groups following the first week. A moderate positive correlation was found between the expression of the VEGF-A gene and protein in all experimental groups $(\mathrm{r}=0.389)$.

Conclusion. Short-term repeated massage may contribute to processes of creation of new and development of already existing vascular networks in the skeletal muscle tissue during increased exercise. (Folia Histochemica et Cytobiologica 2015, Vol. 53, No. 2, 145-151)
\end{abstract}

Key words: rat; physical exercise; massage; skeletal muscle; VEGF-A

\section{Introduction}

The overloading of skeletal muscles is one of the most common causes of injury among professional and amateurs of sports disciplines in which running

Correspondence address: $\mathrm{Ch}$. Kobierzycki, M.D., Ph.D.

Department of Histology and Embryology

Wroclaw Medical University

Chalubinskiego St. 6a, 50-368 Wroclaw

tel.: +48 7178416 82, fax: +48 717840082

e-mail: ch.kobierzycki@gmail.com makes up a significant component of the physical activity [1]. The cause of such overloading is often inadequate preparation for exercise, including lack of warm-up, overly intense exercise in relation to fitness level (insufficient training), aggravation of muscle fiber regeneration after previous overloading, past injuries and the scars caused by them, or the failure of adaptive mechanisms given the increased exercise load. Overload may occur when there is an insufficient supply of oxygenated blood during muscular work, resulting from an underdeveloped network of blood vessels relative to what is needed during intense 
exercise. The process of adaptation occurs in muscle fibers as a result of physical training includes formation of new blood vessels, which leads to increased distribution of blood in the muscles subjected to the load [2]. In many experiments, angiogenesis was observed in response to intense exercise [3-5]. The most important element in the initiation of this process is increased expression of VEGF (vascular endothelial growth factor A), which stimulates endothelial cells proliferation and formation of new blood vessels [6-10]. These phenomena may lead to the subsequent activation of functional adaptation processes, including increases in muscle efficiency.

One widely used treatment in biological renewal, which has been implicated both in the preparing of the body for the physical effort as well as in restoring performance afterward, is massage [11-16]. It is also used as a therapeutic agent in recovering from injuries, and recently has been considered as an important factor in skeletal muscle regeneration. Massage, applied in the form of nonspecific mechanical stimuli (e.g. muscle deformation in the direction transverse to the course of the muscle fibers), can stimulate mechanisms of mechanotransduction and cause many cellular responses in muscle fibers, including the expression of growth factors [17-19]. For instance, massage of muscle tendons while undergoing long-term, systematic running exercise resulted in increased expression of proangiogenic factors' genes, such as $V E G F-A, F G F-2$, and $C D 34$ [20]. Moreover, massage can improve blood circulation in tissues and stimulate reconstruction of microcirculation resulting in the improvement of knee ligaments repair processes following injury [21].

The aim of the present study was to investigate whether muscle massage performed before and during muscular work (running exercise) has an impact on the expression level of the main angiogenic factor, VEGF-A, in the examined rat muscle fibers.

\section{Material and methods}

Animals and experimental design. The study was conducted on 75 ten-month-old Buffalo strain rats housed in identical conditions in the Animal Research Section of the Department of Pathomorphology at Wroclaw Medical University. All rats were subjected to running training on an Exer-3R running track (Columbus Instruments, Columbus, $\mathrm{OH}$, USA) five days per week for ten weeks. The daily duration of training was 10 minutes on the first day, and increased by 5 minutes each day, reaching 30 minutes at the end of the first week. Running was carried out at a speed of $0.3 \mathrm{~m} / \mathrm{s}$ in the first week and subsequently increased to $0.5 \mathrm{~m} / \mathrm{s}$ in the remaining period of the training.
The animals were randomly divided into three groups of 25 rats each. Massage consisted of spiral movements along the flexor digitorum brevis muscle at the plantar surface of $1 \mathrm{~cm}^{2}$ for 5 minutes on each rear extremity [20]. In the first group (PM, the premassaged group), massage was performed five times a week for 3 weeks before the running training. In the second group (M, the massaged group), massage was applied five times a week 10 min before exercise and only during running training. In the third study group ( $\mathrm{C}$, the control group), the massage was omitted. In order to standardize the massage procedure, an algometer device (Digital Algometer Pain Diagnostic Gage, Wagner Instruments, Greenwich, CT, USA) of $0.5 \mathrm{~cm}^{2}$ area with a constant compression power of $9.81 \mathrm{~N}(1 \mathrm{~kg})$ was used.

Euthanasia and sample collection. The study was approved by the Local Ethical Committee No. 1 for Animal Experiments in Wroclaw (decision no. 5/2011). All rats were subjected to anesthesia using ketamine ( $10 \mathrm{mg} / \mathrm{kg}$ body weight) and sacrificed by decapitation. Tissue material for the study was sampled from 5 following rats from each experimental group at the end of weeks 1, 3, 5, 7, and 10 of the experiment. Tissue samples collected from the flexor digitorum brevis muscle of each rear extremity were fixed in $10 \%$ buffered formalin and embedded in paraffin, as well as fixed in liquid nitrogen and stored at $-80^{\circ} \mathrm{C}$ in a deep freezer. Paraffin sections were utilized for histological assessment ( $7 \mu \mathrm{m}$-thick sections) and immunohistochemical evaluation ( $4 \mu \mathrm{m}$-thick sections) under an optical light microscope (BX41 Olympus, Tokyo, Japan), whereas fresh frozen tissues were utilized for the molecular studies - real-time PCR and Western blot.

Immunohistochemistry (IHC). Immunohistochemical reactions were performed with use of an Autostainer Link48 automated staining platform (Dako, Glostrup, Denmark) to ensure constant reaction conditions. Deparaffinization, rehydration, and retrieval of the antigens were achieved by boiling in Target Retrieval Solution (Dako) for $20 \mathrm{~min}$ at $97^{\circ} \mathrm{C}$ using Pre-Treatment Link Platform (Dako). Muscle sections were incubated for 5 min using EnVision FLEX Peroxidase-Blocking Reagent (Dako) to block the activity of endogenous peroxidase. The sections were then incubated for $20 \mathrm{~min}$ at room temperature (RT) with primary antibody directed against VEGF-A (rabbit anti-rat, Antibodies-Online, Aachen, Germany) diluted with EnVision FLEX Antibody Diluent with background reducing component (Dako). Subsequently, sections were incubated for $20 \mathrm{~min}$ at RT with secondary goat anti-rabbit antibodies conjugated to horseradish peroxidase (EnVision/HRP, Dako) and then incubated for $10 \mathrm{~min}$ at RT with 3,3'-diaminobenzidine tetrachlorohydrate (DAB; Dako) which resulted in brown reaction's product. Finally, the slides were counterstained with EnVision $^{\mathrm{TM}}$ FLEX Hematoxylin (Dako) dehydrated in increasing concentrations of alcohol $(70 \%, 96 \%$, and 99.8\%) and xylene, and mounted using SUB-X Mounting 
Medium (Dako). All reagents and equipment apart from the primary antibody against VEGF-A were obtained from Dako, Glostrup, Denmark.

Real-time PCR. Total RNA was isolated from 75 muscle fragments of all rats using RNeasy Fibrous Mini Kit (Qiagen, Hilden, Germany) in line with the manufacturers' recommendations. Reverse transcription reactions were processed using the High-Capacity cDNA Reverse Transcription Kits (Applied Biosystems, Carlsbad, CA, USA). The relative mRNA expression of VEGF-A was evaluated by real-time PCR using the 7900 Fast-Real Time PCR System (Life Technologies, Carlsbad, CA, USA). Real-time PCRs were performed on 384-well plates (Life Technologies) at a set time and temperature, including polymerase activation at $50^{\circ} \mathrm{C}$ for $2 \mathrm{~min}$, preliminary denaturation at $94^{\circ} \mathrm{C}$ for $10 \mathrm{~min}$, denaturation at $94^{\circ} \mathrm{C}$ for $15 \mathrm{~s}$, annealing of primers, and synthesis at $60^{\circ} \mathrm{C}$ at $1 \mathrm{~min}$ for 45 cycles. The primers and probes used in the reactions for VEGF-A (Rn01511601_m1) and for GAPDH (Rn99999916_s1) of TaqMan system were obtained from Life Technologies. Standardization of the results was based on the expression of the reference gene $G A P D H$.

Western blot. Frozen tissue samples of 75 muscles fragments of all rats were homogenized in RIPA lysis buffer [50 mM Tris-Cl pH 8.0, $150 \mathrm{mM} \mathrm{NaCl}, 0.1 \%$ SDS, 1\% Igepal CA-630 (Sigma-Aldrich, St. Louis, MO, USA)], 0.5\% sodium deoxycholate (Sigma-Aldrich), a cocktail of protease inhibitors (Sigma-Aldrich), and 0.5 mM PMSF (Sigma-Aldrich). Protein concentration was measured using the BCA technique (Thermo Scientific, Waltham, MA, USA) with a NanoDrop 1000 spectrophotometer (Thermo Scientific). The tissue extracts were mixed with a sample buffer $[250 \mathrm{mM}$ TRIS pH 6.8, $40 \%$ glycerol, $20 \%$ (v/v) $\beta$-mercaptoethanol, $100 \mathrm{mM}$ DTT, $0.33 \mathrm{mg} / \mathrm{mL}$ bromophenol blue, $8 \mathrm{SDS}]$ and denatured for $10 \mathrm{~min}$ at $95^{\circ} \mathrm{C}$. Equal amounts of protein $(30 \mu \mathrm{g}$ per lane) were separated by electrophoresis according to Laemmli (Laemmli 1970) on a $12 \%$ gel in a Mini Protean 3 apparatus (BioRad, Hercules, CA, USA). Subsequently, the proteins were electrophoretically transferred to a PVDF-P membrane (Immobilon P, Millipore, Darmstadt, Germany) and nonspecific binding sites were blocked using $3 \%$ bovine serum albumin (BSA) in TBST buffer. The amount of applied protein was controlled by staining the total protein on the membrane using Ponceau S (Sigma-Aldrich). The expression of VEGF-A was detected using specific monoclonal anti-VEGF-A antibodies (Dako). Incubation was conducted for $18 \mathrm{~h}$ at $4^{\circ} \mathrm{C}$ with gentle shaking in a solution of the antibody diluted 1:200 in 1\% BSA, in 0.4\% TBSTX (TBS + Triton $\mathrm{x}-100)$. Following incubation, the membrane was washed three times with $0.4 \%$ TBSTX buffer and then incubated for $1 \mathrm{~h}$ with goat anti-mouse antibody conjugated with horseradish peroxidase (HRP) (1:3000, Jackson Immunoresearch, West Grove, PA, USA). Detection was conducted using a chemiluminescent substrate (Luminata ${ }^{\mathrm{TM}}$ Crescendo Western HRP Substrate, Millipore), and the results were documented for exposure times ranging from $2 \mathrm{~s}$ to $30 \mathrm{~min}$ in a Chemi-Doc ${ }^{\mathrm{TM}}$ MP Imaging System (BioRad). The resulting bands were estimated by densitometric quantitative analysis of proteins and normalized to beta tubulin levels (ab 6046, Abcam, Cambridge, UK).

Statistical analysis. Statistical analysis was carried out using Prism 5.0 software (GraphPad, La Jolla, CA, USA). For testing the differences between the PM, M, and C groups, an unpaired $t$-test and analysis of variance (twoway ANOVA) were used, with the Bonferroni correction to counteract the problem of multiple comparisons. The Spearman correlation test was conducted to analyze the existing relationships. Results were considered statistically significant for $\mathrm{p}<0.05$.

\section{Results}

Following the first week of running exercise, a statistically significant $(\mathrm{p}<0.05)$ increase of the VEGF-A mRNA was found in the skeletal muscle of rats massaged for three weeks prior to the exercise (the PM group), as compared to the control group (C), in which no massage was performed (Figure 1A). However, no statistically significant difference was noted in this period between the group of animals massaged during exercise (M) and groups PM and C.

In the further part of the experiment, a gradual increase in the expression of VEGF-A mRNA occurred also in group $\mathrm{M}$. This trend was maintained throughout the whole experiment and reached the statistical significance in comparison to groups PM and $\mathrm{C}$ after 7 and 10 weeks of the experiment (Figure $1 \mathrm{~B})$. In turn, the level of expression of $V E G F-A$ mRNA in the PM group was mostly constant, decreasing slightly between weeks 5 and 7 and showing a slight upward trend between weeks 7 and 10 of the training (Figure 1B). In group $\mathrm{C}$, the level of $V E G F-A$ mRNA remained almost constant during the whole experiment (Figure 1B).

The expression of VEGF-A on the protein level also showed an increase after the first week of running exercise in both the PM and $\mathrm{M}$ groups. These changes were statistically significant in relation to the $C$ group (Figure 2A, B). Furthermore, a statistically significant increase in the levels of VEGF-A protein was found in group $\mathrm{M}$, as compared to group $\mathrm{C}$, from week 1 to week 7 of the experiment, with particular intensity after weeks 3 and 5, and persisted up to week 7 (Figure 2C). A mean positive correlation between the VEGF-A gene expression and the VEGF-A protein levels was also demonstrated in all rats, independently 

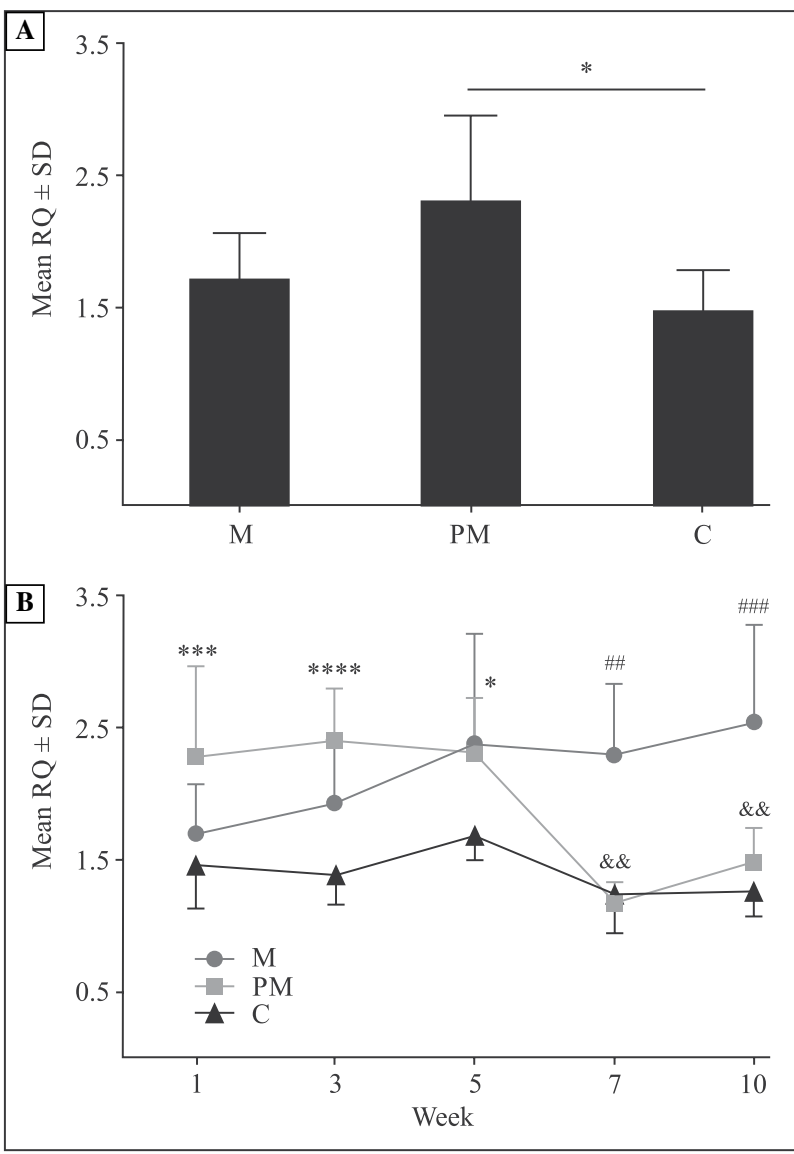

Figure 1. Relative levels (RQ) of $V E G F-A$ mRNA in rat flexor digitorum brevis muscles from the massaged (M), premassaged (PM), and control (C) groups in week 1 of the experiment. Statistical differences are shown between the PM and $\mathrm{C}$ groups ( $\mathrm{p} \mathrm{p}<0.05$; Unpaired $t$-test). Relative level of $V E G F-A$ mRNA was determined as described in Material and methods. (A) Differences in mRNA $V E G F-A$ between the groups - statistically significant differences were observed between $\mathrm{C}$ and PM ( ${ }^{*} \mathrm{p}<0.05$; ${ }^{* *} \mathrm{p}<0.01$; $\left.{ }^{* * * *} \mathrm{p}<0.0001\right), \mathrm{C}$ and $\mathrm{M}\left({ }^{\# \#} \mathrm{p}<0.01 ;{ }^{\# \# \#} \mathrm{p}<0.001\right), \mathrm{M}$ and PM $\left({ }^{\&} \mathrm{p}<0.01\right)$ (Bonferroni multiple comparison test) $(\mathbf{B})$

of the experimental group assignment $(\mathrm{r}=0.389$, $\mathrm{p}<0.001$ ) (Figure 3).

Immunohistochemical technique allowed to determine the cellular localization of VEGF-A, confirming cytoplasmic presence of this protein in skeletal muscle fibers in all 3 groups of rats (Figure 4). Intensity of VEGF-A expression in control group was apparently weaker comparing to PM and M group. In massaged groups expression was not observed in all fibers, suggesting unspecific character of initiating stimulus.

\section{Discussion}

Analyzing the role of massage in sports and in biological renewal in the context of its widespread use raises

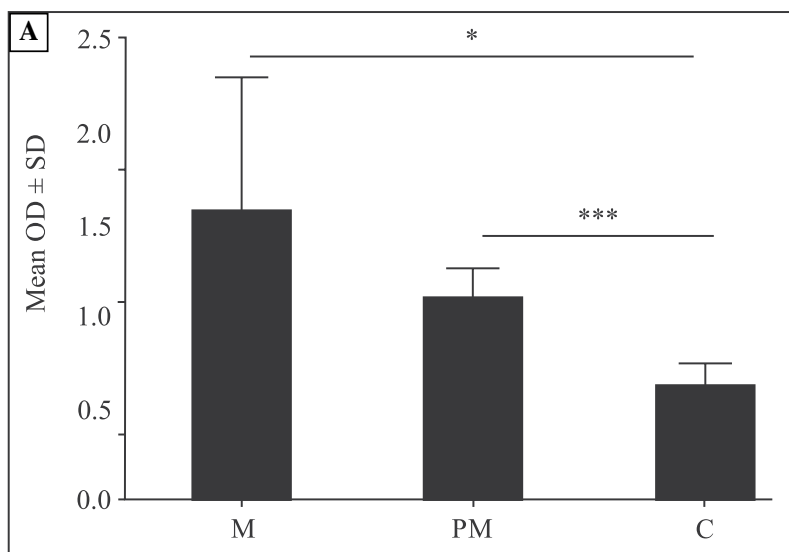

\begin{tabular}{llllllll}
\hline B & M & M & PM & PM & C & C
\end{tabular}
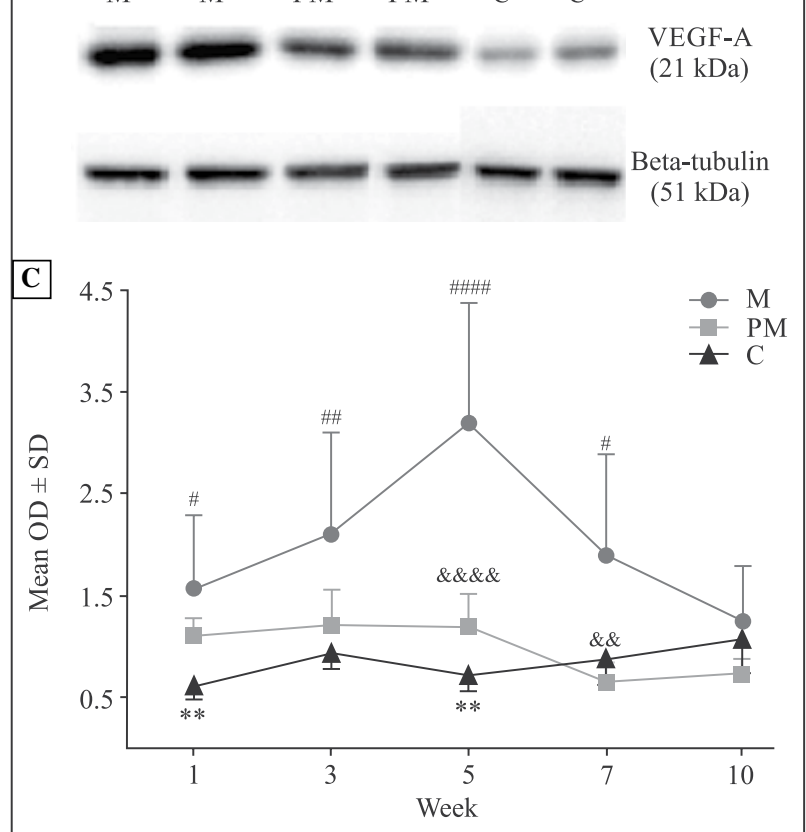

Figure 2. Optical density (OD) of VEGF-A protein expression in rat flexor digitorum brevis muscles of the massaged (M), premassaged (PM), and control (C) groups after 1 week of the running training. Analysis was conducted by Western blotting technique as described in Material and methods. Statistically significant differences between groups are shown $\left({ }^{*} \mathrm{p}<0.05\right.$; *** $\mathrm{p}<0.001$; Unpaired $t$-test) (A). Bands corresponding to the $21-\mathrm{kDa}$ isoform of VEGF-A obtained from muscle sampled in week 5 of the experiment from the massaged (M), premassaged (PM), and control (C) groups (B). Differences between examined study groups. Significant differences were observed between $\mathrm{C}$ and PM ( $\left.{ }^{* *} \mathrm{p}<0.01\right), \mathrm{C}$ and $\mathrm{M}\left({ }^{*} \mathrm{p}<0.05\right.$;

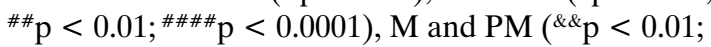
$\left.{ }^{\& \& \&} \mathrm{p}<0.0001\right)$ (Bonferroni multiple comparison test) $(\mathbf{C})$

the question of whether the effects of massage can go beyond the subjective benefits felt by athletes, which have been only minimally corroborated by objective and reliable tests. Conducted experiments have shown that adaptive changes occur in the muscles as a result 


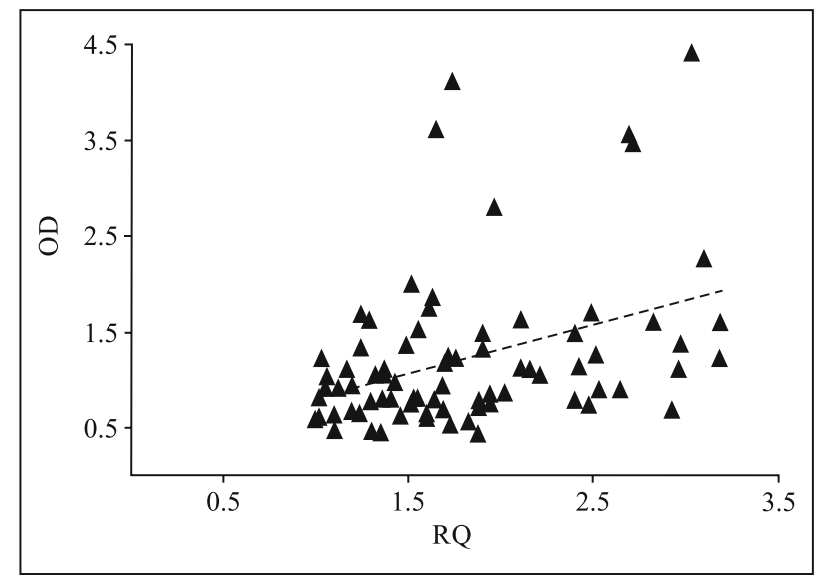

Figure 3. Correlation of mRNA relative expression (RQ) of the $V E G F-A$ gene and optical density (OD) of VEGF-A protein expression (Western blotting) in rat flexor digitorum brevis muscles from all examined cases $(\mathrm{r}=0.389$, $\mathrm{p}<0.001$; Spearman correlation test)

of physical training, and have been evidenced by the formation of new blood vessels and the expansion of already existing ones in a form of network [22]. One of the main markers of this process is increased VEGF expression, indicating endothelial cells proliferation during new blood vessels formation $[6,23]$.

Our results have shown a significant increase of the $V E G F-A$ gene expression in the PM group after the first week of running exercise, whereas predominant growth in the $\mathrm{M}$ group was found in the later part of the experiment. Increases in the expression on the protein level were observed in both massaged groups after the first week of training. A mean positive correlation was shown between the expression of the $V E G F-A$ gene and protein in all groups.

This may indicate that the three weeks of massage prior to physical exercise stimulated the expression of VEGF- $A$ mRNA being a factor initiating formation of new blood vessels. This notion is supported by the fact that in the later phase of the experiment a gradual increase in the expression of $V E G F-A$ mRNA was observed in the $\mathrm{M}$ group. This trend was maintained for the entire duration of the experiment and was statistically significant comparing to the control group. Moreover, expression of $V E G F-A$ mRNA in the PM group evidently decreased after $5^{\text {th }}$ week and slightly increased after $7^{\text {th }}$ and $10^{\text {th }}$ week of exercise. Similar changes were not observed in $\mathrm{C}$ group. This indicates that the physical load at the specific level used in the experiment did not increase the expression of $V E G F-A$ mRNA in the absence of massage.

On the protein level, an increase in the expression of VEGF-A following the first week of exercise in both
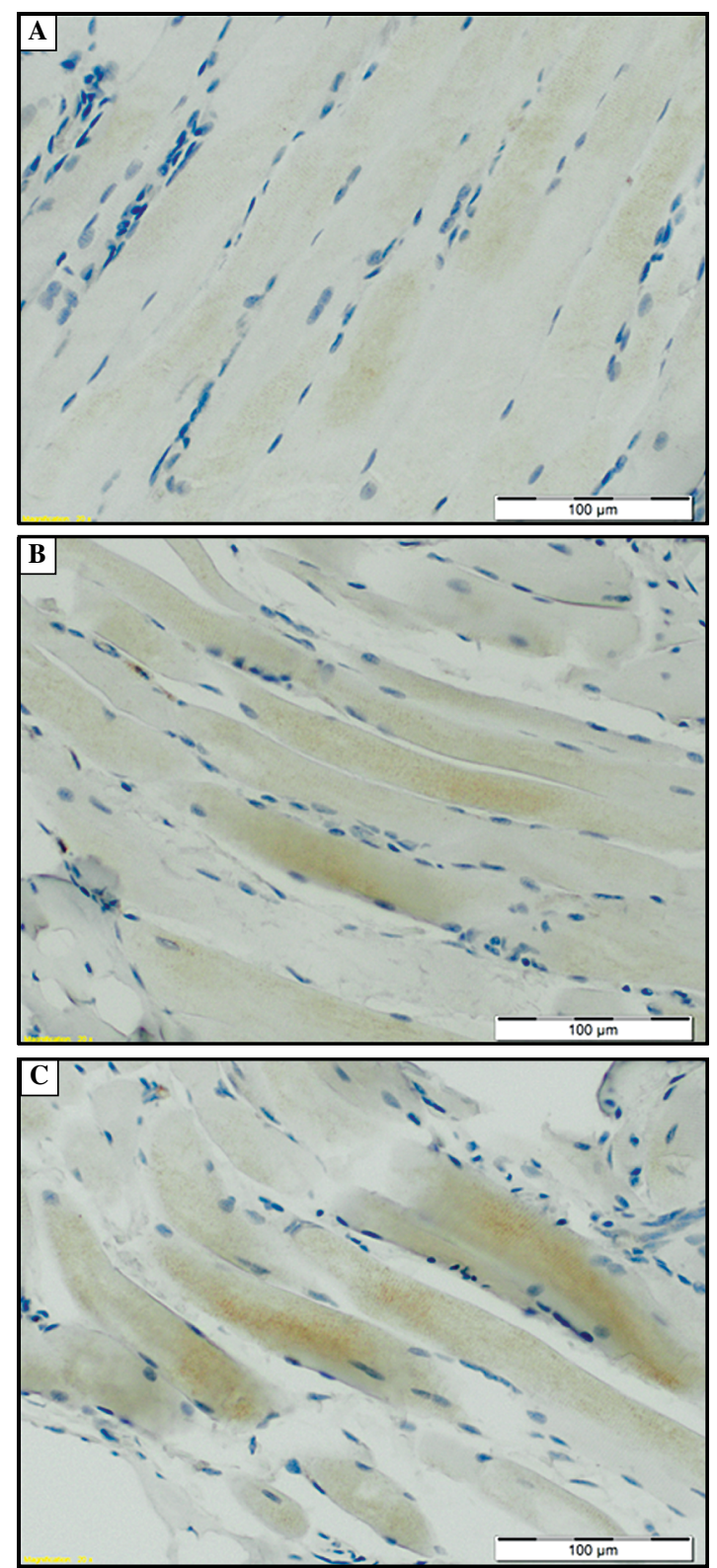

Figure 4. Immunohistochemical sarcoplasmatic expression of VEGF-A protein: low (A), medium (B), and high (C) intensity of immunoreactivity in flexor digitorum brevis muscles from the control (C), premassaged (PM), and massaged (M) group, respectively

the PM and M groups was observed. These changes were statistically significant compared to the C group. This confirms previous observations that massage stimulates the formation of new blood vessels, especially when it is performed during exercise. This can be clearly observed over the full duration of the experiment in the form of the statistically significant increase in the expression of VGEF-A in group $\mathrm{M}$, as compared to group C. Clearly visible raising trend of expression starts already after 1 st week, with a sud- 
den increase after $3^{\text {rd }}$ and $5^{\text {th }}$ week of training. At the same time, a mean positive correlation between the expression of VEGF-A mRNA and protein level was found, pointing to the coherence of achieved results.

The potential effects of massage may be associated with a mechanism based on the response of skeletal muscle fibers to this nonspecific mechanical stimulation through intracellular signals that cause quantitative and qualitative changes in gene expression [24]. Confirmation of such a mechanism might be provided by our previous observation of the increased expression of the $V E G F-A, C D 34$, and $F G F-2$ genes, in massaged tendons of rats during long-term, systematic running exercise [20]. Our present results seem to confirm that the above mechanism could also occur in skeletal muscle fibers, suggesting that massage, a mechanical factor, takes part in the cellular responses inducing angiogenesis. It should be emphasized that massage is a mechanical stimulus not ordinarily met by the muscle, and for this reason its effect is nonspecific; it thus causes more intense reaction than in the case of agents that act for longer periods of time.

\section{Conclusions}

Our results may indicate that the effect of massage goes beyond the subjective benefits of its use experienced by athletes. The statistically significant increase in the expression of VEGF-A in massaged groups of rats, suggests that short-term, repeated massage, synergistically to exercise, may contribute to processes of development and adaptation changes in skeletal muscle tissue. The effect of this may be the creation of new and development of already existing vascular networks in the muscle, and may indirectly contribute to an increase in muscular efficiency, and potentially to regeneration.

\section{Acknowledgments}

This study was partly supported by the National Science Centre Grant no. NN 404002839. The authors declare that there is no conflict of interest.

\section{References}

1. Beiner JM, Joki P. Muscle contusion injuries: current treatment options.J Am Acad Orthop Surg. 2001;9:227-237. PMID: 11476532.

2. Gute D, Fraga C, Laughlin MH, Amann JF. Regional changes in capillary supply in skeletal muscle of high-intensity endurance-trained rats. J Appl Physiol. 1996;81:619-626. PMID: 8872626.

3. Lloyd PG, Prior BM, Yang HT, Terjung RL. Angiogenic growth factor expression in rat skeletal muscle in response to exercise training. Am J Physiol Heart Circ Physiol. 2003;284:1668-1678. doi: 10.1152/ajpheart.00743.2002.
4. Richardson RS, Wagner H, Mudaliar SR et al. Exercise adaptation attenuates VEGF gene expression in human skeletal muscle. Am J Physiol Heart Circ Physiol. 2000;279:772-778. PMID: 10924077.

5. Olfert IM, Breen EC, Mathieu-Costello O, Wagner PD. Skeletal muscle capillarity and angiogenic mRNA levels after exercise training in normoxia and chronic hypoxia. $J$ Appl Physiol. 2001;91:1176-1184. PMID: 11509513.

6. Wagner PD. The critical role of VEGF in skeletal muscle angiogenesis and blood flow. Biochem Soc Trans. 2011;39:1556-1559. doi: 10.1042/BST20110646.

7. Birot OJG, Koulmann N, Peinnequin A, Bigard XA. Exercise-induced expression of vascular endothelial growth factor mRNA in rat skeletal muscle is dependent on fibre type. J Physiol. 2003;552:213-221. doi: 10.1186/1475-2840-7-13.

8. Jensen L, Schjerling P, Hellsten Y. Regulation of VEGF and bFGF mRNA expression and other proliferative compounds in skeletal muscle cells. Angiogenesis. 2004;7:255-267. doi: 10.1007/s10456-004-4184-4.

9. Gavin TP, Wagner PD. Effect of short-term exercise training on angiogenic growth factor gene response in rats. $J \mathrm{Appl}$ Physiol. 2001;90:1219-1226. PMID: 11247917.

10. Roudier E, Gineste Ch, Wazna A et al. Angio-adaptation in unloaded skeletal muscle: new insights into an early and muscle type-specific dynamic process. J Physiol. 2010;588:4579-4591. doi: 10.1113 /jphysiol.2010.193243.

11. Best TM, Hunter R, Wilcox A, Haq F. Effectiveness of sports massage for recovery of skeletal muscle from strenuous exercise. Clin J Sport Med. 2008;18:446-460. doi: $\underline{10.1097 /}$ JSM.0b013e31818837a1.

12. Zainuddin Z, Newton M, Sacco P, Nosaka K. Effects of massage on delayed-onset muscle soreness, swelling and recovery of muscle function. $J$ Athl Train. 2005;40:174-180. PMID: 16284637.

13. Mori $\mathrm{H}$, Ohsawa $\mathrm{H}$, Tanaka $\mathrm{TH}$ et al. Effect of massage on blood flow and muscle fatigue following isometric lumbar exercise. Med Sci Monit. 2004;10:173-178. PMID: 15114265.

14. Jonhagen S, Ackermann P, Eriksson T, Saartok T, Renström PA. Sports massage after eccentric exercise. Am J Sports Med. 2004;32:1499-1503. doi: 10.1177/0363546503262196.

15. Butterfield TA, Zhao Y, Agarwal S, Haq F, Best TM. Cyclic compressive loading facilitates recovery after eccentric exercise. Med Sci Sports Exerc. 2008;40:1289-1296. doi: 10.1249/ MSS.0b013e31816c4e12.

16. Hart JM, Swanik CB, Tierney RT. Effects of sport massage on limb girth and discomfort associated with eccentric exercise. J Athl Train. 2005;40:181-185. PMID: 16284638.

17. MacKenna D, Summerour SR, Villarreal FJ. Role of mechanical factors in modulating cardiac fibroblast function and extracellular matrix synthesis. Cardiovasc Res. 2000;46:257-263. doi: 10.1016/S0008-6363(00)00030-4.

18. Kjaer M. Role of extracellular matrix in adaptation of tendon and skeletal muscle to mechanical loading. Physiol Rev. 2004;84:649-698. doi: 10.1152/physrev.00031.2003.

19. Chiquet M, Gelman L, Lutz R, Maier S. From mechanotransduction to extracellular matrix gene expression in fibroblasts. Biochim Biophys Acta. 2009;1793:911-920. doi: 10.1016/i. bbamcr.2009.01.012.

20. Andrzejewski W, Kassolik K, Dziegiel P et al. Effects of synergistic massage and physical exercise on the expression of angiogenic markers in rat tendons. Biomed Res Int. 2014;2014:878095. doi: 10.1155/2014/878095.

21. Loghmani MT, Warden SJ. Instrument-assisted cross-fiber massage increases tissue perfusion and alters microvascular 
morphology in the vicinity of healing knee ligaments. $B M C$ Complement Altern Med. 2013;13:240. doi: 10.1186/1472-688213-240.

22. Gustaffson T, Kraus WE. Exercise-induced angiogenesis -related growth and transcription factors on skeletal muscle and their modification in muscle pathology. Front Biosci. 2001;6:75-89. PMID: 15730338.
23. Richardson RS, Wagner H, Mudaliar SR et al. Human VEGF gene expression in skeletal muscle: The effect of acute normoxic and hypoxic exercise. Am J Physiol Heart Circ Physiol. 1999;277:2247-2252. PMID: 10600843.

24. Costa A, Dalloul H, Hegyesi H et al. Impact of repeated bouts of eccentric exercise on myogenic gene expression. Eur J Appl Physiol. 2007;101:427-436. doi: 10.1007/s00421-007-0510-z.

Submitted: 23 June, 2015

Accepted after reviews: 2 July, 2015 Available as AoP: 6 July, 2015 